\title{
« Les exigences linguistiques dans le recrutement d'un clergé pour l'Ouest canadien, 1818-1920 »
}

\section{Robert Painchaud}

Volume 42, 1975

URI : https://id.erudit.org/iderudit/1007236ar

DOI : https://doi.org/10.7202/1007236ar

Aller au sommaire du numéro

Éditeur(s)

Les Éditions Historia Ecclesiæ Catholicæ Canadensis Inc.

ISSN

0318-6172 (imprimé)

1927-7067 (numérique)

Découvrir la revue

Citer cet article

Painchaud, R. (1975). « Les exigences linguistiques dans le recrutement d'un clergé pour l'Ouest canadien, 1818-1920 ». Sessions d'étude - Société canadienne d'histoire de l'Église catholique, 42, 43-64. https://doi.org/10.7202/1007236ar

Tous droits réservés ㄷ Les Éditions Historia Ecclesiæ Catholicæ Canadensis Inc., 1976
Ce document est protégé par la loi sur le droit d'auteur. L'utilisation des services d'Érudit (y compris la reproduction) est assujettie à sa politique d'utilisation que vous pouvez consulter en ligne.

https://apropos.erudit.org/fr/usagers/politique-dutilisation/ 


\section{"Les exigences linguistiques dans le recrutement d'un clergé pour l'Ouest canadien, 1818-1920 »}

Le chercheur qui dépouille les fonds d'archives des évêchés de l'Ouest canadien ne peut s'empêcher d'être saisi par le grand nombre de documents qui portent sur la nécessité de connaître plus d'une langue, soit pour être admis dans les rangs du clergé de tel ou tel diocèse, soit encore pour remplir efficacement les devoirs du ministère soit enfin pour répondre aux besoins d'une population catholique de plus en plus hétérogène. La question est d'autant plus importante qu'elle pose le problème d'une Église foncièrement française œuvrant dans un milieu où les droits des groupes français et catholiques furent contestés et abolis. Aussi, les chefs de l'Église, depuis la fondation d'une mission permanente à la Rivière-Rouge en 1818, jusqu'aux changements apportés dans le diocèse de SaintBoniface dans les années 1910 , ont-ils cherché à réconcilier leur rôle de chefs de file de la collectivité française avec celui de pasteurs de l'Église universelle. C'est dans ce contexte que cette communication vise à faire un premier bilan des démarches faites par quatre évêques de l'Ouest — Provencher, Taché, Langevin, et Legal - pour obtenir des individus ou des communautés religieuses aptes à nouer des liens entre les catholiques de nationalités diverses ${ }^{1}$.

\section{Mor Joseph-Norbert Provencher}

Avant même son départ de Kamouraska pour la lointaine mission de la Rivière-Rouge, le jeune abbé Joseph-Norbert Provencher doutait de ses capacités pour ce travail, vu son * défaut de langage

*** Une bourse de doctorat du Conseil des Arts du Canada a grandement facilité une recherche approfondie et continue au cours des deux dernières années. Encore faut-il remercier les archevêques et évêques de l'Ouest canadien qui ont permis la consultation de leurs fonds d'archives.

1 S'il n'est pas question dans ce travail de Mgr Vital-J. Grandin ou de Mgr Albert Pascal, c'est que nous n'avons pas eu l'occasion de dépouiller leurs archives. 
du pays, au moins de l'anglais 2 ». Aussi, après une année dans la colonie, pouvait-il souhaiter que $\mathrm{M}^{\mathrm{gr}}$ Plessis lui envoie de nouvelles recrues qui «sussent l'anglais, langue que nous entendons tous trois un peu et que nous ne parlons guère ${ }^{3}$ ». Il ajoutait toutefois qu'il serait encore plus désirable que l'un des deux missionnaires attendus «passât l'hiver au lac des Deux Montagnes pour y étudier le sauvage et transcrire beaucoup de choses que nous n'aurons pas facilement ici. Il apprendra dans trois ou quatre mois là plus qu'ici en deux ans parce que nous n'avons rien sur cette langue ${ }^{4}$ ". Évêque, $\mathrm{M}^{\mathrm{gr}}$ Provencher ne cessa pas de se préoccuper de la question indienne, et surtout de l'acquisition de membres du clergé en mesure d'apprendre la langue algonquine. Ce n'est qu'avec l'arrivée de l'abbé Georges-Antoine Belcourt à Saint-Boniface en 1831 qu'il put alors compter sur un sujet qui se consacrerait exclusivement à l'évangélisation des Indiens. Quelques années plus tard toutefois, au grand regret de $\mathrm{M}^{\mathrm{gr}}$ Provencher, l'abbé Belcourt, bien désillusionné, quittait Saint-Boniface, emportant avec lui le dictionnaire qu'il avait soigneusement élaboré. Il revint bientôt au pays, mais le quitta de nouveau en 1847 suite à des démêlés qu'il eût avec la Compagnie de la Baie d'Hudson dans l'affaire du commerce libre ${ }^{5}$. Par conséquent, $\mathrm{M}^{\mathrm{gr}}$ Provencher, trente ans après son arrivée dans l'Ouest, cherchait toujours un sujet qui pourrait exercer le ministère auprès des populations indiennes. L'expectative de l'arrivée de l'abbé Jean-Baptiste Proulx le réjouit, car « un prêtre, qui vaut trois par les langues qu'il parle, doit être admis avec joie s, écrivait-il à $\mathbf{M}^{\mathbf{g r}}$ Bourget ${ }^{6}$. Malheureusement, ses attentes furent trompées, de sorte qu'il appartiendrait à son successeur, $M^{\text {gr }}$ Alexandre Taché, de voir à assurer la continuité dans ce domaine.

2 Bulletin de la Société Historique de Saint-Boniface, Vol. III, Lettres de Monseigneur Joseph-Norbert Provencher, Saint-Boniface (Imprimerie du Manitoba), 1913, p. 6. Kamouraska, 15 mars 1818, Provencher à Mgr J.O. Plessis.

3 Ibid., p. 40. Saint-Boniface de la Rivière-Rouge, 27 juillet 1819, Provencher à Mgr Plessis. Ses compagnons étaient l'abbé Sévère Dumoulin et Guillaume Edge, séminariste.

4 Ibid..

5 Voir Donatien Frémont, M ${ }^{g r}$ Provencher et son temps, Winnipeg (Éditions de La Liberté), passim. Aussi la biographie dans le Dictionnaire biographique du Canada, Vol. X, PUL (1972), pp. 49-51.

6 Les Cloches de Saint-Boniface, Vol. XX, no. 4 (avril 1921), pp. 69-72. Saint-Boniface, 13 juin 1848, Provencher à [Bourget]. 
Si une connaissance des langues indigènes venait en priorité dans les projets de $\mathbf{M}^{\text {gr }}$ Provencher, c'est qu'il y avait peu ou pas de catholiques de langue anglaise dans la colonie. Ayant conclu une entente en 1834 pour avoir un maitre capable d'enseigner l'anglais, il écrivit à $\mathrm{M}^{\mathrm{gr}}$ Signay que dans le choix d'un nouveau missionnaire il ne fallait pas sacrifier d'autres bonnes qualités au manque de connaissance de l'anglais ?. Ce n'est que dans les années 1840 que se fit sentir le besoin d'un clergé instruit en anglais. Les désirs qu'avait Provencher d'améliorer l'enseignement dans ses écoles, joints à l'activité grandissante des ministres protestants, le poussèrent à faire des demandes réitérées pour obtenir des religieux ou des religieuses. L'évêque de Dubuque lui offrit trois bons sujets, mais parce que ccs religicuses ne connaissaient pas le français, l'évêque de Saint-Boniface préférait obtenir des sœurs canadiennes ${ }^{8}$.

Les quatre sœurs Grises venues fonder une œuvre d'éducation dans la colonie dès 1844 savaient l'anglais. Mais $\mathrm{M}^{\text {gr }}$ Provencher, un mois après leur arrivée dans le pays, notait d'autres besoins. Il en fit part à $\mathbf{M}^{\mathrm{gr}}$ Signay :

J'ai demandé à Mgr de Dubuc (sic) une fille capable de tenir une bonne école anglaise... Elle viendrait l'été prochain si j'en avais besoin. Plusieurs attendaient l'arrivée des Sœurs pour savoir si elles tiendraient une école anglaise. Il y en aura probablement bien d'autres s'il y avait des Sœurs Grises capables à cette besogne. J'insisterais plus pour en avoir mais il n'y en avait pas à mon départ 9 .

Quatre années plus tard, il renouvelait sa demande auprès de $\mathbf{M}^{\mathrm{gr}}$ Bourget, "pour avoir des institutrices capables de faire une bonne école anglaise et montrer plusieurs branches d'éducation qu'il n'est pas nécessaire d'apprendre aux enfans (sic) de nos cultivateurs ${ }^{10}$ 》. Il s'agissait d'attirer les enfants des bourgeois qui étaient prêts à payer cher, et de permettre ainsi aux sœurs de vivre. $\mathrm{M}^{\mathrm{gr}}$ Proven-

7 Bulletin..., p. 139. Rivière-Rouge, 16 juillet 1834, Provencher à Mgr Signay.

8 Archives de la chancelerie de l'archevêché de Montréal (ACAM), dossier du Diocèse de Saint-Boniface, 1836-1850. Québec, 26 octobre- 1843, Provencher à $\mathbf{M g r}$ Bourget.

9 Bulletin..., p. 237. St-Boniface de la Rivière-Rouge, 29 juillet 1844, Provencher à $\mathbf{M g r}$ Signay.

$10 A C A M$, Diocèse de ..., [s.1.], 19 juillet 1848, Provencher à $\mathbf{M g r}$ Bourget. Aussi dans LCSB, Vol. XX, no 6 (juin 1921), pp. 114-115. 
cher ne se plaignait pas de celles qui se trouvaient déjà en fonction. « Elles sont toutes des bonnes filles, disait-il, mais qui ne répondent pas aux besoins du pays, quelques unes par défaut d'éducation et toutes par le manque de la langue anglaise 11 ». Deux événements stimulèrent ses démarches : d'abord, l'arrivée d'une quinzaine de familles irlandaises catholiques ${ }^{12}$, puis la fermeture de l'école des filles anglaises à la suite de la mort du ministre $\mathrm{McCallum}^{13}$. Deux ans plus tard, l'école anglaise des sœurs n'allait pas mal, mais on $y$ trouvait peu de pensionnaires et les bourgeois du pays n'y avaient pas encore envoyé leurs filles ${ }^{14}$.

Il fallait quelque chose de plus. Par conséquent, $\mathbf{M}^{\mathrm{gr}}$ Provencher forma le dessein d'avoir des Frères de St-Viateur à condition "qu'ils parlassent Anglais, [car] il n'y a point de maître d'école ici ${ }^{15}$ ». $\mathrm{M}^{\mathrm{gr}}$ Taché, nommé coadjuteur du vieil évêque et assurant par son sacre la présence continue des Oblats au Nord-Ouest, adressa une demande dans le même sens à $M^{\text {gr }}$ Bourget, vu «l'état d'infériorité où se trouvent les Catholiques de la Rivière-Rouge par rapport à l'éducation ${ }^{16}$ ». Il souffrait à la vue de métis et de sauvages éduqués par les protestants et possédant un degré bien passable d'instruction, "tandis que parmi les nôtres il y en a si peu que ce n'est véritablement pas la peine d'en parler ». Qui plus est, on accusait l'Église d'être responsable de cet état de chose. II suppliait $\mathrm{M}^{\mathrm{gr}}$ de Montréal "[d']envoyer l'été prochain, deux frères bien formés et en état d'enseigner bien le Français et l'Anglais ${ }^{17}$ 》. Un choix judicieux des sujets s'imposait, car il était question non seulement d'instruire les enfants catholiques, mais encore d'inciter les païens à confier leurs enfants aux Frères. Hélas!, $\mathrm{M}^{\mathrm{gr}}$ Provencher mourut avant que ne fut réalisé ce projet des Frères. Aussi, en décrivant à $\mathbf{M}^{\mathrm{gr}}$ Bourget la mort du premier évêque de Saint-Boniface survenue le 7 juin 1853, l'abbé Louis-François Laflèche lui rappelait l'importance de l'envoi des Frères. Une ample besogne les at-

11 Ibid.

12 LCSB, Vol. X , no. 6 (juin 1921), pp. 115-117. Saint-Boniface, 29 novembre 1848, Provencher à Mgr Bourget.

13 LCSB, Vol. XX, no 8 (août 1921), pp. 152-154. Saint-Boniface, 29 novembre 1849, Provencher à Mgr Bourget.

$14 L C S B$, Vol. XX, no. 10 (octobre 1921), pp. 192-194. Saint-Boniface, 15 juillet 1851, Provencher à $\mathrm{Mgr}$ Bourget.

15 ACAM, Diocèse de Saint-Boniface (1851-1871). Saint-Boniface, 6 juillet 1852, Provencher à Mgr [Bourget].

16 Ibid., Rivière-aux-Brochets, 22 juillet 1852, Taché à Mgr [Bourget].

17 Ibid. 
tendait dans la colonie, surtout celle de l'instruction des garçons que les Sours n'arrivaient pas à maîtriser. «Il faut au moins, écrivait le futur évêque de Trois-Rivières, qu'il y en ait un capable d'enseigner l'anglais parce que plusieurs de nos enfans (sic) catholiques sont obligés de fréquenter des écoles protestantes, parce que nous ne pouvons pas leur faire enseigner l'anglais... ${ }^{18}$ 》. Au mois de novembre suivant, l'abbé Laflèche regrettait beaucoup de voir que des Frères pour les écoles n'étaient pas encore arrivés dans la colonie. On les attendait plus que jamais, afin de pouvoir multiplier les écoles et les églises et opposer au mal engendré par une "légion de ministres 》 qui élevaient eux aussi des écoles et des églises un «remède de même nature ». Il fallait nécessairement qu'au moins un, et préférablement deux des Frères, puissent enseigner l'anglais. De plus, un père parlant l'anglais serait fort bien accueilli, et répondrait à l'un «des besoins les plus urgents » de la mission, celui d'exercer le ministère auprès d'une «quarantaine de familles irlandaises et catholiques desservies bien misérablement ${ }^{19}$ ». Un an plus tard, $\mathrm{M}^{\mathrm{gr}}$ Taché embrassait trois frères de la Doctrine chrétienne à leur arrivée à Saint-Boniface. Étaient-ils bilingues ? Les documents ne le disent pas ${ }^{20}$.

En résumé, à l'époque des premières fondations de l'Église dans l'Ouest, $\mathrm{M}^{\mathrm{gr}}$ Provencher chercha d'abord à former un clergé pour évangéliser les peuples indigènes en demandant des auxiliaires qui soient prêts à apprendre les langues indiennes. Pour l'anglais, ce n'est qu'à partir du moment où se dressa une rivalité entre catholiques et protestants qu'il devint nécessaire, voire urgent, d'assurer des écoles dans lesquelles l'enseignement de l'anglais occuperait une place aussi importante que les autres matières scolaires. L'usage en était toutefois restreint à la colonie de la Rivière-Rouge, les missions lointaines n'étant pas encore affectées.

18 Ibid., Saint-Boniface de la Rivière-Rouge, 3 juillet 1853, Laflèche à $\mathrm{Mgr}$ [Bourget].

19 Ibid., Saint-Boniface de la Rivière-Rouge, 4 novembre 1853, Lafleche à Mgr Bourget.

20 Les Frères quittèrent toutefois la Rivière-Rouge en juillet 1860 . Voir Dom Paul Benoît, Vie de Mgr Taché, Vol. I, Montréal (Beauchemin, 1904), p. 419. Mgr Taché regretta beaucoup ce départ: "C'est un malheur et un grand. Je le regrette amèrement, mais je n'ai pu le détourner. Je vous remercie beaucoup de nous avoir cédé $M$. Oram; il fera très bien pour l'anglais. ...nos écoles enfin allaient si bien.» $A C A M$, Diocèse de St-Boniface (1851-1871), Rivière-Rouge, 27 juillet 1860, Taché à Mgr Bourget. William Henry Oram repartit en septembre 1862. 


\section{II. $M^{g r}$ Alexandre-Antonin Taché}

Durant l'épiscopat de $\mathrm{M}^{\mathrm{gr}}$ Taché, la population catholique dans l'Ouest canadien passa d'environ 6,000 en 1870 à plus de 12,000 en 1881 , et à près de 20,000 en $1891^{21}$. Fait plus important à remarquer toutefois, c'est qu'à la mort du premier archevêque de Saint-Boniface en 1894, on comptait, en plus des catholiques d'origine française, métisse ou indienne, des groupes importants de catholiques d'origine flamande, allemande et irlandaise. Ce mélange de nationalités allait poser de sérieux problèmes au premier métropolitain de l'Ouest.

Une de ses préoccupations constantes fut celle des écoles. Alors qu'il assistait au chapitre général des Oblats en France en 1867, il reçut la «bien pénible nouvelle » que le Frère, qui à St-Boniface était chargé de la classe anglaise, avait déserté son poste. $\mathbf{M}^{\mathrm{gr}}$ de Montréal pourrait-il lui trouver «quelqu'un de sûr pour enseigner la langue anglaise ? ${ }^{22} \gg$ Il faudra attendre l'arrivée des Pères Jésuites à Saint-Boniface en 1885 pour que soit assuré l'enseignement de l'anglais au Collège. Entre temps, d'autres maisons d'enseignement réclamaient des sujets bilingues. Les Sœurs des Saints Noms de Jésus et de Marie prirent le relève de l'Académie Ste-Marie de Winnipeg en 1874 suite au départ des Sœurs Grises ${ }^{23}$. Restait à trouver une communauté religieuse qui prendrait en main l'instruction des jeunes garçons. Dans ses nombreux voyages au Québec, le Père Albert Lacombe, à la demande expresse de $\mathbf{M}^{\mathrm{gr}}$ Taché, lança un appel infructueux à plusieurs congrégations d'hommes. D'Europe, l'infatigable voyageur Oblat rapportait en 1879 qu'il avait vu à Lyon le Supérieur Général des frères Maristes, mais qu'il était impossible d'avoir des sujets de cette congrégation, qui, pourtant, aurait si bien convenu à $\mathbf{M}^{\mathrm{gr}}$ Taché «puisqu'elle a des sujets anglais 24 ». Le Père Lacombe entendait encore s'adresser aux frères du Sacré-Cour qui avaient des maisons en Amérique, ainsi qu'aux frères Marianistes de New York, et enfin, aux frères de Lamennais qui avaient des maisons en Angleterre et en Irlande ${ }^{25}$. On

21 Voir les recensements de 1870 (Manitoba), de 1881, et de 1891.

22 ACAM, Diocèse de ... (1851-1871). Paris, 20 août 1867, Taché à Mgr [Bourget].

23 Benoît, op. cit., Vol. II, pp. 245-246.

24 Archives de l'archidiocèse de Saint-Boniface (AASB), Fonds Taché, Autun [France], 28 juillet 1879, Lacombe à Taché.

25 Ibid. 
peut imaginer avec quel soulagement on accueillit l'année suivante, après six années de recherches, trois Frères de la Congrégation de Marie venus assumer la direction de cette école rattachée à la paroisse mixte de Ste-Marie de Winnipeg ${ }^{26}$.

Même en dehors de la région urbaine de Saint-Boniface-Winnipeg, les besoins de l'anglais se faisaient sentir. L'abbé Louis-Raymond Giroux, curé de Ste-Anne des Chênes, s'inquiétait de l'avenir de son école en 1874 , ne sachant pas s'il réussirait à trouver une personne pour la tenir ouverte. "Pour bien faire, disait-il, il nous faudrait quelqu'un qui pût enseigner l'anglais vu qu'il y a des familles anglaises qui y envoient leurs enfants... ${ }^{27} \gg$. De même, lors de l'établissement de la colonie de Canadiens français rapatriés sur les bords de la Rivière Rouge en 1876-77, le Père Lacombe indiquait à $\mathrm{M}^{\mathrm{gr}}$ Taché qu'il faudrait trouver un prêtre, coûte que coûte, mais aussi un maître d'école pour le nouveau centre. "Si vous me permettez, écrivait-il, j'en engagerai un ici [à Montréal], connaissant et pouvant enseigner les deux langues ${ }^{28}$ ". Fait à noter, lorsque l'on envoya des Sœurs Grises à Ste-Anne (1883) et des Sœurs des Saints Noms à St-Pierre-Jolys (1886), on se contenta d'institutrices canadiennes dont la connaissance de l'anglais ne devait pas être très grande. $\mathbf{M}^{\mathrm{gr}}$ Taché et $\mathbf{M}^{\mathrm{gr}}$ Grandin de St-Albert choisirent cependant de s'adresser aux Fidèles Compagnes de Jésus, congrégation dont la maison mère se trouvait en Bretagne mais qui dirigeait des écoles en Angleterre, pour la fondation de couvents-pensionnats à Brandon, Prince-Albert et St-Laurent de Grandin, là où la population était composée en partie d'éléments anglophones ${ }^{29}$. Ainsi, dès les débuts de la colonisation au lendemain de 1870, il devenait manifeste que l'Église aurait à choisir soigneusement ses auxiliaires.

Qu'en était-il du service paroissial ? Des indices nous portent à croire que même dans les paroisses à majorité française, telles SteAnne des Chênes ou Ste-Agathe, le clergé devait pouvoir au moins entendre les confessions en anglais, quitte à prêcher uniquement

26 Benoît, op. cit.,. Vol. II, pp. 386-387.

27 AASB, Fonds Taché, Ste-Anne des Chênes, 18 mai 1874, Giroux à Mgr Taché.

28 Ibid., Montréal, 23 mars 1877, Lacombe à Mgr Taché.

29 Benoît, op. cit., Vol. II, p. 423. Aussi dans Adrien-Gabriel Morice, o.m.i., Histoire de l'Église Catholique dans l'Ouest canadien (1659-1915), Vol. III, Saint-Boniface (Chez l'Auteur), 1922, pp. 37, 58-59. 
en français ${ }^{30}$. De toute façon, la marche du mouvement de colonisation dans l'Ouest favorisait l'élément non-français. Or, tout en insistant pour obtenir un plus grand nombre de colons d'origine française soit du Québec, soit des États-Unis, soit enfin de l'Europe, $M^{\mathrm{gr}}$ Taché entendait les besoins de son diocèse. Ainsi, en 1882 , voyant la nécessité de prêtres pour desservir trois localités nouvelles, il demandait à $\mathbf{M}^{\mathrm{gr}}$ Fabre de lui rendre « le plus grand service » en lui cédant " un ou deux prêtres, surtout s'ils parlaient l'Anglais et le Français ${ }^{31}$ ». Trois ans auparavant, il avait accueilli avec joie dans son diocèse le Père Théobald Bitsche, membre de la Congrégation du Précieux-Sang de Rome, qui avait accompagné à la Montagne Pembina des familles allemandes et irlandaises établies antérieurement à Guelph, en Ontario. Ces colons, de parents alsaciens et lorrains et dont plusieurs n'avaient "point oublié le français », vinrent grossir les rangs de la petite colonie canadiennefrançaise de St-Léon ${ }^{32}$. Non sans heurts cependant. Le Père Bitsche, versé en quatre langues, eut bientôt de la peine à unir ses paroissiens; certains Canadiens voulaient avoir un prêtre canadien, et expulser les Allemands ${ }^{33}$. Dix ans plus tard, à la mort du curé Bitsche, c'était au tour des paroissiens allemands de réclamer un prêtre connaissant leur langue. Dom Paul Benoît, Chanoine Régulier de l'Immaculée Conception et fondateur de Notre-Dame de Lourdes et de St-Claude, posa le problème à $\mathrm{M}^{\mathrm{gr}}$ Taché dans les termes suivants :

Les habitants de St-Léon m'ont présenté, le jour de la clôture de leur retraite, une adresse pour me demander le plus tôt possible un prêtre résidant toute la semaine, et pour demander que ce prêtre fut le $\mathbf{P}$. Agnèce. J'ai écrit aussitôt à mon Supérieur pour le prier de nous envoyer immédiatement, s'il le pouvait, quelques jeunes religieux. Je pense qu'il $\mathrm{y}$ aura, vers la fin du présent mois, un prêtre à demeure à St-Léon. Quant à leur donner le P. Agnèce, les canadiens

30 À défaut de registres de lettres de Mgr Taché qui nous auraient permis de saisir sa pensée en ce domaine et de suivre les démarches qu'il fit pour s'assurer le services de prêtres et de religieux possédant des aptitudes linguistiques, il faut donc s'en remettre à la volumineuse correspondance conservée aux Archives de l'Archidiocèse de Saint-Boniface et y glaner des traces de sa ligne de conduite.

$31 A C A M$, Diocèse de Saint-Boniface (1879-1896). St-Boniface, 14 septembre 1882, Taché à Mgr Fabre.

32 AASB, Fonds Taché. St-Boniface, 4 août 1879, Bitsche à Mgr Taché.

33 Archives paroissiales de St-Norbert. St-Léon, 28 mars 1882, Bitsche à [l'abbé Joseph-Norbert] Ritchot. 
seuls le désirent, les allemands (ils sont une quinzaine de familles) préfèrent le $P$. Marie-Antoine, qui est un alsacien et parle leur langue. Je crois que le mieux sera de [leur] envoyer les deux pères à tour de rôle ou ensemble. Il y a beaucoup de rivalité dans cette paroisse entre canadiens et allemands 34 .

Le Père Agnèce, CRIC, était nommé curé quelques mois plus tard, alors que le Père Marie-Antoine Straub, CRIC, originaire de l'Alsace, prenait la paroisse en main en 1898. Quoiqu'il en soit, StLéon conserva sa prépondérance française ${ }^{35}$.

À mesure qu'augmentait le flot d'immigration étrangère, $\mathrm{M}^{\mathrm{gr}}$ Taché voyait crôtre les instances pour un clergé bilingue. Son grand vicaire, le Père Joseph Allard, o.m.i., lui demandait où trouver un prêtre parlant le Polonais pour les quelque 50 à 60 familles polonaises qui en avaient grandement besoin ${ }^{36}$. D'autre part, le Père Jules Decorby, en visite à Langenburg dans le territoire de l'Assiniboia, où il rencontra une douzaine de familles arrivées de Gallicie depuis quelques mois seulement et parlant l'Allemand, conseillait à son évêque d'envoyer là un prêtre allemand qui pourrait desservir en même temps une autre colonie en voie de formation aux environs de Lanshut ${ }^{37}$. De Régina, l'abbé Joseph Caron rapportait pour sa part que les Allemands de Balgonie étaient très anxieux d'avoir un missionnaire appartenant à leur nationalité. Il proposait en outre que l'on envoie le jeune scholastique Alphonse Lemieux, après son ordination, dire la messe à Moose Jaw et dans les missions plus à l'Ouest, mais il avouait que ce dernier ne savait que très peu d'anglais ${ }^{38}$. Enfin, plus près de Saint-Boniface, des difficultés surgirent dans les paroisses de St-Alphonse, Bruxelles, et Mariapolis, où se mêlaient Canadiens, Belges Wallons et Belges

34 AASB, Fonds Taché. Notre-Dame de Lourdes, 5 août 1892, Dom Benoît à Mgr Taché.

35 Lors d'un recensement paroissial en 1903, St-Léon, y compris la mission de Somerset, contenait $\mathbf{7 6}$ familles canadiennes-françaises, 4 familles métisses, 4 familles irlandaises, 3 familles françaises, et seulement 8 familles allemandes. Voir Marie Anna-A. Roy, La Montagne Pembina au temps des colons, Winnipeg (Canadian Publishers), 1970, p. 39.

36 AASB, Fonds Taché. St-Boniface, 18 octobre 1889, J. Allard, o.m.i., à $\mathrm{Mgr}$ Taché.

37 Ibid., Langenburg [Assiniboia], 10 juillet '1891, J.-A. Decorby, o.m.i., à $\mathbf{M g r}$ Taché.

38 Ibid., Régina, 12 janvier 1892, J. Caron à Mgr Taché. 
Flamands. On voulait des prêtres capables de réconcilier les divergences de vues qui les divisaient ${ }^{39}$.

Comment se comporta $\mathrm{M}^{\mathrm{gr}}$ Taché devant toutes ces réclamations? Pour y répondre, il faut supposer qu'il ne prévoyait guère une invasion d'immigrants de nationalités étrangères comme il s'en produisit à l'heure de la grande immigration d'après 1896 . On peut croire, par conséquent, que pour lui, le pays conserverait, devait conserver, sa dualité anglaise et française, tout en assimilant les nouveaux arrivés dans l'une ou l'autre des deux sociétés co-fondatrices. Pas surprenant donc qu'il cherchât à s'entourer d'un clergé foncièrement français, mais pouvant converser en anglais ${ }^{40}$. Ainsi, Edmond Faiseaux, délégué par le gouvernement canadien en 189192 pour remplacer l'abbé Jean Gaire dans une tournée en Belgique au profit de la colonisation de l'Ouest canadien, communiqua à $\mathrm{M}^{\mathrm{gr}}$ Taché deux demandes de prêtres désireux de se rendre au Canada, l'assurant qu'il les avait prévenus «que Votre Grandeur tenait à ce que ses prêtres sachent l'anglais ${ }^{41}$ ». Toutefois, lorsque l'un de ces prêtres, l'abbé Albert Leuret, se présenta soudainement dans son diocèse craignant peut-être d'être refusé parce qu'il savait «peu d'anglais », $\mathrm{M}^{\mathrm{gr}}$ Taché accepta de le mettre à l'essai. Peu après la mort de l'archevêque, l'abbé Leuret, en fonction à Willow Bunch, reconnaissait qu'il ne savait pas «suffisamment l'anglais pour exer-

39 Les Canadiens s'établirent les premiers dans cette région dans les années 1880 , alors que les Belges commencèrent à y affluer dans les premières années de la décennie suivante.

40 Il est un incident qu'il vaut la peine de noter par rapport à l'utilisation des deux langues principales courantes dans l'Ouest à l'époque. À l'été de 1890 , donc au moment où se posaient les questions scolaires tant au Manitoha que dans les Territoires du Nord-Ouest, Jules-Paul Tardivel, en tournée dans l'Ouest, abordait un «sujet délicat» suite à son passage à Qu'appelle où l'on donnait l'instruction aux jeunes sauvages en anglais à l'école industrielle. Il écrivait que Mgr Taché avait cédé « devant les exigences des sauvages eux-mêmes. Et le motif qui les a fait céder, c'est uniquement le désir de sauver les âmes de ces pauvres païens 》 Voir «Lettres de voyage » (suite et fin) dans La Vérité, 9 août 1890. Malheureusement, ni Mgr Taché ni aucun de ses proches collaborateurs ne releva cette affirmation du journaliste québécois. Était-ce parce que d'autres observations de Tardivel retinrent d'abord leur attention ou encore parce que son analyse était juste? Avait-on songé à franciser et les peuples indiens et les groupes d'immigrants européens, politique qui se serait avérée inapplicable?

41 AASB, Fonds Taché. Bincke [Belgique], 8 février 1892, Edmond Faiseaux à Mgr Taché. 
cer mon ministère en cette langue ${ }^{42} \gg$. Par contre, un autre prêtre, l'abbé A. Delecourt, bien recommandé par $\mathbf{M}^{\mathrm{gr}}$ Racine et l'abbé Proulx suite à une rencontre au séminaire canadien à Rome, et parlant le français, l'anglais, et l'allemand, ne semble pas s'être rendu dans l'Ouest ${ }^{43}$. $\mathrm{M}^{\mathrm{gr}}$ Taché garda-t-il le premier parce qu'il ne put obtenir le second?

Bref, $\mathrm{M}^{\mathrm{gr}}$ Taché se contenta des prêtres qu'il accueillit à l'occasion et, en tout temps, fit appel à sa communauté pour lui fournir des ouvriers apostoliques. C'est un Oblat, le Père A. Pagé, qui s'occupa des Hongrois établis non loin de Lebret en 188944 . Au Manitoba, $\mathrm{M}^{\mathrm{gr}}$ Taché instruisit l'abbé $\mathrm{F}$. Turcotte de visiter périodiquement les catholiques parlant l'anglais à Manitou et qui avaient fait des instances «pour être visités par quelqu'un qui parle leur langue 45 ». À St-Eustache, paroisse située à l'ouest de Winnipeg, le curé Arcade Martin, dans un rapport soumis à l'autorité épiscopale et contenant des renseignements pour l'année 1894, donnait comme population catholique 264 Canadiens français, 63 Canadiens anglais, et 490 métis français et anglais. Sans doute, exerçait-il son ministère dans les deux langues, quoiqu'il estimait que «la nationalité qui finira par s'emparer de St-Eustache est la nationalité canadienne française car c'est elle qui fait plus de progrès 46 ». Encore faut-il dire que l'on chargea un prêtre séoulier parlant le français et le flamand d'aller desservir la paroisse de Bruxelles (Manitoba) en 1893, parce que $M^{\text {gr }}$ Taché et ses collaborateurs à l'œuvre de la colonisation étaient persuadés qu'une immigration belge considérable devait bientôt affluer vers les plaines de l'Ouest.

Il n'en était pas de même pour l'immigration allemande, hongroise, polonaise, et même irlandaise qui, le croyait-on à l'époque,

42 Ibid., Trainnon, par Loury [Loiret, France], 10 mai 1892, Albert Leuret à Mgr Taché; ibid., Willow-Bunch, 21 (?) août 1894, Leuret au Père [Allard ?]. Mgr Taché.

43 Ibid., Rome [Séminaire français], 8 février 1892, A. Delecourt à

44 Ibid., Lebret, 8 février 1889, J.-P. Magnan à Mgr Taché.

45 AASB, Fonds Benoît-[Guéret], «Lettres de Mgr Taché », St-Boniface, 12 juillet 1892, Mgr Taché à Dom Benoît.

46 Archives de l'Archidiocèse de Winnipeg ( $A A W)$, Dossier St-Eustache. St-Eustache, $1^{\mathrm{er}}$ février 1895, A.-M. Martin à Mgr Langevin. Le curé Martin s'expliqua en disant que la grande majorité des familles métisses étaient «très arriérées », la plupart ne possédant «pas même un pouce de terrain». Il croyait toutefois que quelques-uns donnaient «de fortes espérances pour l'avenir.» 
n'arriverait pas à dépasser en nombre la population catholique de langue française. Pour le moment, on pouvait toujours compter sur les Jésuites pour s'occuper d'une paroisse anglaise telle Portage la Prairie, ou encore sur les Chanoines Réguliers de l'Immaculée Conception pour trouver un père parlant l'allemand, mais surtout sur les Oblats qui continuaient à fournir la majorité des prêtres. En 1894, année du décès de $\mathbf{M}^{\mathrm{gr}}$ Taché, seul le diocèse de Saint-Boniface possédait autant de séculiers que de religieux. Ailleurs, les Oblats, pour la plupart venus de l'Europe, dirigeaient toutes les paroisses et missions. Mais alors que par le passé il n'avait pas été nécessaire pour eux de connaître l'anglais, en dehors de quelques villes établies, voilà que le besoin se faisait sentir dans des centres naissants, comme en témoigne le Père L. Lebret, envoyé de PrinceAlbert à Battleford, "pour aider le P. [Victor] Pineau, qui ne parle point anglais; or, il y a, ici, un certain nombre de familles irlandaises, qui ne comprennent point le français, de là, la raison de mon transvasement, momentané ou autrement ${ }^{47}$.

Somme toute, $\mathrm{M}^{\mathrm{gr}}$ Taché visa à s'entourer d'un clergé conforme à la société dualiste reconnue en 1870 par l'Acte du Manitoba et revendiquée par la suite par la population française de l'Ouest. De plus, tout en souhaitant la venue de renforts catholiques, préférablement tirés des pays français, il reconnut les obligations qu'il avait envers les autres immigrants de même foi. Il ne fut jamais question, cependant, de chercher à recruter systématiquement des prêtres ou des religieux en mesure de desservir les fidèles dans des langues autres que le français ou l'anglais. Il n'en fut pas ainsi pour $\mathbf{M}^{\mathrm{gr}}$ Adélard Langevin ou pour $\mathrm{M}^{\mathrm{gr}}$ Émile Legal, comme on le verra.

\section{III. $M^{\text {gr }}$ Adélard Langevin et $M^{\text {gr }}$ Emile Legal}

Les dernières années du $\mathrm{XIX}^{\mathrm{e}}$ siècle apportèrent des changements dans la vie de l'Église de l'Ouest canadien. L'essor économique qui débuta vers 1896 , coïncidant avec la formation du ministère de Wilfrid Laurier à Ottawa, eut pour effet de faciliter le peuplement rapide des terres vacantes des plaines de l'Ouest. C'est à $\mathbf{M}^{\mathrm{gr}}$ Adélard Langevin, le successeur de $\mathbf{M}^{\mathrm{gr}}$ Taché au siège archiépiscopal de Saint-Boniface en 1895 , et à $\mathbf{M}^{\text {gr }}$ Émile Legal, coadjuteur de $\mathrm{M}^{\text {gr }}$ Grandin en 1897 et évêque de St-Albert en

47 AASB, Fonds Langevin. Battleford, 1 er décembre 1894, L. Lebret, o.m.i., au Père Allard. 
1902, que reviendraient la lourde charge de présider aux destinées de l'Église durant les années mouvementées qui conduisirent au démembrement, dès 1911-12, d'une structure composée presque exclusivement d'ecclésiastiques français. Poussés par la vague d'immigration étrangère à s'engager dans une course aux clochers afin d'assurer la présence de l'Église dans les fondations nouvelles, ils cherchèrent en même temps à poursuivre des objectifs précis : la restauration des droits scolaires abolis dans les années 1890; le maintien de la primauté de l'élément français à la tête des forces catholiques; la création de maisons d'enseignements et d'œuvres diverses pour servir la population catholique; et, enfin, le rassemblement des fidèles de toutes nationalités sous les drapeaux de l'Église persécutée, croyait-on, par des phalanges libérales. C'est donc dans ce contexte qu'il faut étudier le recrutement du clergé fait par ces deux évêques Oblats.

Peu de temps après sa consécration épiscopale, $\mathrm{M}^{\mathrm{gr}}$ Langevin eût à se pencher sur la situation de ses diocésains de langue flamande. En effet, dans une supplique que lui adressèrent des 'notables' de St-Alphonse, on le priait de ne pas leur enlever l'abbé Telesphore Campeau, curé depuis dix ans, invoquant son rôle indispensable dans le mouvement d'immigration belge vers le Manitoba ainsi que ses affinités d'intérêts avec les colons. Les pétitionnaires ajoutaient : " la langue anglaise et flamande que possède notre Curé sont indispensables pour la colonie $»$; par conséquent, son départ signifierait pour eux «la destruction de notre colonie 48 ». Aussi, la nomination de l'abbé Noël Perquis comme curé de cette paroisse et des missions avoisinantes ne répondit pas aux besoins des fidèles qui revinrent à la charge en 1896 . Cette fois, $M^{\mathrm{gr}}$ Langevin, dans une lettre adressée aux "bons fidèles de la paroisse catholique de St-Alphonse », leur répondit qu'il n'avait pas les moyens d'entretenir à ses frais un prêtre parlant cette langue ${ }^{49}$. L'année suivante, toutefois, toujours à la demande des colons flamands, l'abbé Gustave Willems, curé de la paroisse belge de Bruxelles, se chargea également de St-Alphonse. Non sans difficultés. Willems, qui prêchait tous les dimanches en français et en flamand, ne plut guère à certains flamands "auprès de qui mon plus grand crime est

48 AASB, Fonds Langevin. St-Alphonse, 20 août 1895, [des 'notables'] à $\mathrm{Mgr}$ Langevin.

49 Ibid. Notre-Dame de Lourdes, 19 juin 1896, Langevin «Aux bons fidèles de la paroisse catholique de St-Alphonse. » 
d'être trop l'ami des Canadiens ${ }^{50}$ ». Lorsqu'il quitta la province peu de temps après, $\mathbf{M}^{\mathrm{gr}}$ Langevin résolut de trouver des prêtres qui pourraient en imposer aux Flamands non seulement par leur connaissance de la langue, mais encore par leur origine. Ainsi, l'abbé Hubert Heynen, natif d'une province flamande, prenait en main la paroisse de Bruxelles, qu'il devait diriger pendant 47 ans ${ }^{31}$. Quant à St-Alphonse, le Père Albert Lacombe, délégué par l'archevêque en 1900 pour traiter de plusieurs questions importantes en Europe, n'arriva pas à conclure une entente avec des Prémontrés de Belgique pour la desserte de cette paroisse ${ }^{52}$. Enfin, en $1904, \mathrm{M}^{\mathrm{gr}}$ Langevin confiait St-Alphonse au Père Charles Deutschler, CRIC, qui, en dépit de sa lenteur à maîtriser la langue et de sa préférence marquée pour les Canadiens de Mariapolis, du moins dans les débuts, y demeura jusqu'à sa mort en 1923 53. De cette manière, $\mathrm{M}^{\mathrm{gr}}$ Langevin régla pour un temps les besoins de ce groupe de colons ${ }^{54}$.

L'exemple des Flamands démontre bien qu'il ne suffisait plus, au tournant du siècle, d'envoyer occasionnellement un prêtre parlant telle ou telle langue dans une paroisse renfermant un groupe minoritaire linguistique quelconque. On revendiquait de plus en

50 Ibid. St-Alphonse, 10 septembre 1898, Willems à Mgr Langevin.

51 Roy, op. cit., pp. 81-98 (passim).

$52 A A S B$, Fonds Benôt -[Guéret], «Lettres de Mgr Langevin, 18951900 ", St-Boniface, $1^{\text {er }}$ août 1900, Langevin à Dom Benoît; AASB, Fonds Langevin, Paris, 28 août 1900, Lacombe à Mgr Langevin; ibid., Bruxelles (Belgique), 3 septembre 1900, Lacombe à Mgr Langevin; ibid., Paris, 27 septembre 1900, Lacombe à Mgr Langevin. Il avait déjà été question de Prémontrés pour desservir les colons belges à la Montagne de la Tortue.

53 Peu après son arrivée au Canada en 1901, le Père Deutschler écrivait à Dom Benoît qu'il était venu par obéissance, et qu'il souhaitait que l'on envoie «quelqu'un, plus capable que moi, pour apprendre le flamand». AASB, Fonds Benoit-[Guéret], «St-Alphonse », St-Alphonse, 7 avril 1902, Deutschler à Dom Benoît.

54 En 1914, Mgr Langevin écrivait au Recteur du Collège Américain, à Louvain en Belgique, afin d'obtenir «des sujets connaissant la langue flamande, et française, et capables d'apprendre aussi l'anglais. »Il disait avoir frappé inutilement à la porte de plusieurs évêchés, depuis cinq à six ans, alors qu'il aurait peut-être dû songer à trouver plutôt des séminaristes. Pour l'instant, il lui fallait au plus vite «un jeune prêtre sachant le Flamand pour remplacer un prêtre Belge vicaire à la cathédrale qui peut me quitter d'un moment à l'autre. Il s'agirait d'organiser, si possible, une paroisse de Flamands à St-Boniface même où il y a près de (200) deux cents familles Belges, " Cette paroisse fut érigée en 1917. Voir AASB, Fonds Langevin, St-Boniface, 4 et 14 février 1914, Langevin au Supérieur du Séminaire Américain [Mgr J.A. De Becker], Louvain, Belgique. 
plus l'envoi de prêtres appartenant à la nationalité des fidèles mêmes, ou encore l'organisation de paroisses nationales contrairement au Droit Canon qui ne permettait que des cadres territoriaux regroupant tous les catholiques. Dans Winnipeg, il se fondait néanmoins des paroisses pour les Polonais (1899), les Allemands (1904), et les Canadiens français (1905) ${ }^{55}$. Des tentatives semblables de regroupement furent opérées dans les autres grandes villes de l'Ouest, alors que dans les régions rurales les paroisses de nationalités mixtes exigeaient des compromis trop souvent très ardus. Un vicaire d'origine allemande expliquait de la façon suivante à $\mathbf{M}^{\mathrm{gr}}$ Langevin en 1908 les difficultés que l'on éprouvait dans la paroisse de Qu'Appelle où se trouvaient 153 Allemands, 115 Canadiens français, et 55 Irlandais ou Anglais :

...to the remark about the Frenchmen (sic), I regret too that I don't know French yet but as I have promised I will learn it as soon as possible. On the other hand the Frenchmen here are not right, to render suspected (sic) the German priest as they have done because he doesn't preach French sermons. What would the Frenchmen have said if the Germans here in the town, who are in the majority, had rendered suspected (sic) the French priest since he doesn't preach in German these two years he is here? Why did he not learn German? 36

En présence de cette situation, l'épiscopat de l'Ouest comprit qu'il leur fallait recruter des prêtres non seulement bilingues, mais multilingues. Ainsi, par exemple, en même temps qu'il proposait aux Missionnaires de la Salette la fondation d'une paroisse française à St-Hubert de la Rollandrie en $1899, \mathrm{M}^{\mathrm{gr}}$ Langevin leur demandait un second père, sachant l'anglais et pouvant apprendre le

35 Déjà en 1892, Mgr Taché recevait une pétition de la part d'une cinquantaine de familles canadiennes-françaises appartenant à la paroisse de Ste-Marie de Winnipeg, dont \& un grand nombre d'entre nous, les femmes surtout, ne comprennent pas du tout ou pas assez l'anglais pour profiter des instructions données dans une langue étrangère à la nôtre aux offices », et qui demandaient que les instructions et les annonces soient faites dans leur langue maternelle au moins à l'une des messes dominicales. On rapportait aussi «l'antipathie » de certains paroissiens à l'égard de l'usage du français à l'église. Voir AASB, Fonds Taché, [1892], (Paroissiens de Winnipeg) à $\mathbf{M g r}$ Taché.

56 Archives de l'Archidiocèse de Régina (AAR), Dossier qu'Appelle, South Qu'Appelle, 11 juin 1908, F.J. Fresen à Mgr Langevin. 
hongrois, pour desservir deux missions voisines ${ }^{57}$. Aussi, dès 1903, $\mathbf{M}^{\mathrm{gr}}$ Langevin demanda à l'abbé Jean Gaire, l'un de ses plus proches collaborateurs à l'œuvre de la colonisation et son représentant en Europe lors de ses fréquents voyages, de ne plus attirer de prêtres français vers son diocèse ${ }^{58}$. Deux ans plus tard, le métropolitain proclamait une nouvelle politique dans les Cloches de Saint-Boniface :

Il n'y a plus guère de place en ce moment dans nos diocèses de l'Ouest pour les prêtres étrangers ne sachant ni l'anglais, ni l'allemand, ni le polonais, ni le hongrois; mais tout prêtre français sera le bienvenu s'il veut fonder une paroisse en pleine prairie vierge en y ammenant des colons. Il y a encore des immensités libres 59.

Paradoxalement, alors que la collectivité française de l'Ouest traversait une période très difficile et souhaitait voir grossir les effectifs français, l'Église se trouva obligée à refuser des prêtres et des religieux qui fuyaient une Europe chambardée par le mouvement anti-clérical. $\mathrm{M}^{\mathrm{gr}}$ Legal répondait à l'un de ces prêtres qu'il ne pouvait lui conseiller de venir au Nord-Ouest, parce que "presque tous les petits postes, qui se fondent, de divers côtés, sont composés de Catholiques de différentes nationalités, et il est nécessaire que le service puisse se faire au moins en français et en anglais \& je suppose que vous ne pourriez remplir ces conditions 60 ». Un dernier exemple suffira pour saisir d'autres nuances de ce problème : en 1911, l'évêque de St-Albert refusait d'une part un prêtre canadienfrançais, du diocèse de Burlington, Vt., faute de poste à lui offrir. alors que, d'autre part, il encourageait un nouveau prêtre, d'âge avancé, à venir voir et juger pour lui-même si les conditions de son diocèse pourraient lui convenir. Il ajouta encore :

J'ai assurément bien besoin de prêtres et surtout de prêtres parlant plusieurs langues. Vous désirez apprendre l'Allemand avant de venir. Je n'y ai pas d'objection si vous pensez que

57 AASB, Fonds Langevin, RL 1, [St-Boniface], 25 mai 1899, Langevin au Père Joseph Vignon, M.S., Supérieur, LaSalette College, Connecticut.

58 AAW, Dossier Grande Clairière, Wauchope, 3 août 1903, Gaire à Mgr Langevin.

59 LCSB, Vol. IV, no. 20 (décembre 1905), p. 306, «Avis au Clergé Français. 》

60 AAE, Fonds Legal, RL IV, p. 450, [St-Albert], 26 février 1904, Mgr Legal à l'abbé Dupeux (Charente). 
cela ne vous prendrait pas trop de temps. Mais puisque vous avez su déjà le Russe passablement, je préférerais que vous preniez plutôt le temps de vous perfectionner dans la connaissance de cette langue. Car nous avons ici, à Edmonton, $\&$ dans les environs, beaucoup de gens de races $\&$ de langues slaves: Russes, Polonais, Ruthènes ? \& en connaissant le Russe vous seriez compris de tous ces gens \& pourriez les comprendre 61.

Il serait facile de continuer cette étude en examinant les démarches faites par l'épiscopat de l'Ouest auprès de nombreuses communautés religieuses, les unes vouées à l'enseignement, les autres dévouées à d'autres œuvres sociales, afin des les intéresser à établir des maisons de leur congrégation dans l'ouest du Canada. Pour l'instant, il nous suffira de dire que leurs sollicitations le plus souvent empressées ont suivi de près la ligne de conduite adoptée dans le cas du clergé paroissial. Au chapitre des écoles, $\mathbf{M}^{\mathrm{gr}}$ Langevin résuma en quelques phrases l'importance de la connaissance et de l'enseignement de l'anglais:

Si nous voulons maintenir nos écoles et sauver le français, il faut absolument tenir compte de l'anglais.

Dura lex, sed lex.

M. Cherrier prépare le programme de diplômes pour les candidats de langue française.

Il $\mathrm{y}$ aura beaucoup de français, mais il y aura une bonne mesure d'anglais! Or, pour préparer les élèves à ces diplômes il est nécessaire de leur enseigner la langue anglaise 62.

C'est à partir de ce sine qua non que les évêques de l'Ouest acceptèrent ou refusèrent des communautés enseignantes qui s'offraient pour ouvrir des écoles paroissiales, ou encore pour prendre en main des écoles publiques. Il n'était plus question toutefois, comme dans les années 1840 , d'attirer chez soi les protestants, mais plutôt de sauver les écoles coûte que coûte. Au supérieur de la communauté des Sœurs de St. Gildas du diocèse de Nantes, Mrr Legal répondait en 1904 qu'il avait accueilli les Sours de Kermaria (en Morbihan) pour tenir des écoles élémentaires, surtout des écoles de campagne, mais que, étant donné leur faible connaissance de

61 Ibid., RL X, pp. 159-160, [St-Albert], 17 avril 1911, Legal à l'abbé Philippe Casgrain (Hereford, Angleterre).

$62 A A S B$, Fonds Langevin, RL 3, St-Boniface, 24 décembre 1900, Langevin à Dom Benoît, 
la langue anglaise, elles s'étaient d'abord confinées à tenir quelques maisons dans le diocèse. Donc, il lui était difficile de donner refuge à une autre communauté dont les membres ignoraient aussi l'anglais ${ }^{63}$. Cette réponse type revient souvent dans les dossiers.

De façon générale, les évêques francophones de l'Ouest canadien auraient souhaité maintenir la prépondérance de l'élément français au sein du clergé. Mais on reconnut assez tôt qu'il était difficile, voire quasi impossible, de trouver suffisamment de prêtres d'esprit et de formation française capables de maîtriser les langues des catholiques appartenant aux autres nationalités. Les expériences vécues à St-Léon dans les années 1880 et à St-Alphonse au cours de la décennie suivante se répétèrent dans le cas des Allemands et des Ruthènes au tournant du siècle, et, plus tard, dans le cas des Irlandais. Il ne suffisait pas de connaître la langue des fidèles pour se faire respecter ou accepter; on exigeait des prêtres de même nationalité. C'est $\mathrm{M}^{\mathrm{gr}}$ Legal qui résuma en peu de mots l'imprudence d'une démarche qui paraîtrait vouloir latiniser les Ruthènes de rite Grec en leur envoyant des prêtres Canadiens ou autres, mais n'appartenant pas à cette nationalité; "On aura beau apprendre lä langue, bâtir des écoles, ..., on ne deviendra jamais des prêtres ruthènes ${ }^{64}$ ». Par la suite, les évêques de la province ecclésiastique de Saint-Boniface s'appliquèrent à trouver des prêtres et des religieux possédant de nettes affinités avec les peuples confiés à leurs soins ${ }^{65}$. Cette politique, il faut le dire, eut pour aboutissement logique et presque inévitable, la fondation d'une Église ukrainienne au Canada, en 1912, sous la direction d'un patriarche, $\mathrm{M}^{\mathrm{gr}}$ Nicétas Budka. En même temps, on remit entre les mains des Pères Bénédictins de Muenster - monastère fondé en 1903, érigé en abbaye en 1911, et en abbaye mullius en 1921 - la desserte de neuf paroisses et missions foncièrement allemandes en Saskatchewan. Faut-il ajouter que les Irlandais en réclamèrent autant, et y arrivèrent avec la nomination d'évêques Irlandais pour les nouveaux

63 AAE, Fonds Legal, RL IV, [St-Albert], 18 janvier 1904, Legal à l'abbé Baschelier, Supérieur, Nantes. Langevin.

64 AASB, Fonds Langevin, St-Albert, 2 juillet 1900, Legal à Mgr

65 Cela n'empêcha pas toutefois des prêtres français de passer du rite latin au rite ruthène. Voir Joseph JEAN, «S.E. Mgr Adélard Langevin, Archevêque de St-Boniface, et les Ukrainiens", dans le Rapport de La Société Canadienne d'Histoire de l'Église Catholique (1944-45), pp. 101-110. 
diocèses de Calgary (1912) et de Winnipeg (1915) ${ }^{66}$. En moins de vingt ans, l'Église de l'Ouest avait été «ethnicisée ».

Les partisans de l'anglicisation de l'Église de l'Ouest, qui entrèrent en contestation avec le clergé français de la région au cours de la période 1896-1915, et qui reprochaient à celui-ci l'encouragement donné à la conservation des langues nationales, poussèrent $\mathrm{M}^{\mathrm{gr}}$ Langevin à justifier sa politique sur la question des langues. Dans un mémoire sur le diocèse de Saint-Boniface, préparé en 1912, le métropolitain expliqua de la façon suivante son appui du système bilingue :

On s'est demandé d'abord pourquoi il y avait si peu de prêtres de langue anglaise dans l'Ouest?

La réponse est bien simple : c'est que, d'abord, il y a eu très peu de catholiques de langue anglaise dans le pays, et qu'ils étaient souvent mêlés comme ils le sont encore en beaucoup d'endroits, soit aux protestants ou à des catholiques en majorité ou en nombre égal mais parlant une autre langue. Alors il fallait et il faut encore avoir des prêtres parlant et l'anglais et une autre langue comme le français ou l'allemand, et l'on sait que les prêtres de langue anglaise ou bien ne savent pas d'autre langue ou bien se prêtent difficilement à ce genre de ministère bilingue.

...

L'Ouest canadien n'est pas un bonum derelictum dont chacun peut s'emparer, ou un pays sauvage ouvert au premier occupant. Nous venons de le dire : tout $\mathrm{y}$ est bien organisé. Nous supposons évidemment, qu'il ne s'agit pas d'imposer la langue anglaise dans les églises des catholiques de langue française, polonaise, allemande, hongroise, et ruthène! $\mathrm{Ce}$ serait une pratique contraire à celle des apôtres qui ont parlé la langue de ceux qu'ils évangélisèrent, et cela, grâces (sic) à un miracle, signe évident de la volonté de Dieu. Ce serait mettre en péril, la foi de nos fidèles, que de leur imposer la langue anglaise, et ils se révolteraient contre cette imposition odieuse.

...

Nous admettons cependant que les colons étrangers apprendront plutôt l'anglais que toute autre langue, et que tôt ou tard ils finiront, sans doute, par ne parler que l'anglais;

66 Voir Robert ChoquetTe, "Adélard Langevin et l'érection de l'archidiocèse de Winnipeg », dans $R H A F$, Vol. 28, no. 2 (septembre 1974), pp. 187-207. Nous ne partageons pas pleinement l'analyse de cet auteur, qui isole le conflit entre les Irlandais et les Canadiens français au lieu de le situer dans le contexte des relations entre toutes les nationalités de l'Ouest. 
mais il s'écoulera du temps, peut-être un demi-siècle et plus, avant qu'ils cessent de parler leur langue. Toute tentative de précipiter cet événement ne pourrait que le retarder et mettrait certainement en danger la foi d'un grand nombre.

Parlons donc clairement. Nous considérons que c'est le devoir des parents et du clergé de veiller à ce que les enfants du pays apprennent la langue anglaise dans les écoles; ce serait faire du tort aux enfants, et ce serait se montrer peu intelligents et peu soucieux du bien social de nos catholiques que de négliger ce devoir. Il faut aussi apprendre aux enfants à respecter et à aimer le noble drapeau britannique en leur rappelant ce que les catholiques lui doivent et aussi ce que ce drapeau doit aux catholiques qui l'ont défendu parfois au prix de leur sang. Mais c'est le droit et le devoir des parents de faire apprendre à leurs enfants la langue des aieux, la langue de la vieille foi catholique 67 .

Ce qu'il faut surtout retenir de ce singulier document, c'est la place secondaire qu'il paraît accorder à l'usage du français pour les fonctions du ministère paroissial. On peut l'expliquer toutefois en disant que $\mathbf{M}^{\mathrm{gr}}$ Langevin, toujours un fin politique, choisit de ne pas trop insister sur la prépondérance de l'élément français au sein de son clergé d'une part, et, d'autre part, misa sur les rapports étroits entre langue et foi. Pourtant, les données rapportées dans Le Canada ecclésiastique pour l'année 1916, illustrent l'influence continue du clergé francophone dans la conduite des ouvres de l'Église au moins dans les diocèses de Saint-Boniface et de Régina. Dans le premier, on retrouve 146 prêtres de langue française appartenant au clergé séculier et régulier, comparativement à 16 Anglais, 9 Polonais, 9 Hollandais, et un Allemand; dans le second. 69 prêtres séculiers ou réguliers sont d'origine française, contre 5 Anglais, 19 Allemands, 6 Polonais, 2 Hollandais, et 2 Hongrois ${ }^{68}$.

67 AASB, Fonds Langevin, [s.1., 1912 ?], " Mémoire sur le diocèse de Saint-Boniface. » Ce document, préparé au dactylographe, n'est pas signé et n'indique pas non plus un destinataire.

68 Voir Le Canada ecclésiastique pour l'année 1916, (Montréal, Librairie Beauchemin), pp. 341-342, 374. Aussi, dans un rapport envoyé au Délégué Apostolique du Canada en 1911, en prévision de l'érection d'un diocèse à Calgary, Mgr Legal fournissait les renseignements suivants sur le clergé qui serait compris dans le nouveau diocèse : 28 Oblats, 3 Pères de Ste-Marie de Tinchebray, 3 Missionnaires du Sacré-Cour (France), un Bénédictin et un Père Prémontré. Tous parlaient le français et l'anglais, et, de plus, sept d'entre eux parlaient l'Allemand, un le Polonais et le Ruthène, et un l'Italien. $A A E$, Fonds Legal, RL X, pp. 458-471, [St-Albert ?], 5 décembre 1911, Legal à Mgr P. Stagni, Délégué Apostolique, Ottawa. 
Chez les religieuses, la proportion de francophones est à peu près la même, mais il existe un nombre significatif de polonaises, d'allemandes, et d'anglaises. Si l'on ajoute à ces statistiques les prêtres, religieux, et religieuses d'origines ethniques diverses ou de rite ruthène œuvrant dans les autres diocèses de l'Ouest, il est évident cependant qu'il s'était opéré une transformation profonde dans la composition du clergé du pays depuis le début des grandes migrations dans les années 1890. D'insistance sur un clergé essentiellement français mais bilingue, $M^{\text {gr }}$ Langevin et ses suffragants en étaient venus d'abord à conclure à la nécessité, et ensuite à reconnaître les avantages, d'un clergé non seulement multilingue mais encore multi-ethnique ${ }^{69}$.

\section{Conclusions}

Trois époques, trois conjonctures, trois politiques. Voilà qui résume le cheminement des évêques de l'Ouest en matière de recrutement du clergé durant ce premier siècle de la vie de l'Église dans cette partie du Canada.

À l'ère missionnaire de $\mathrm{M}^{\mathrm{gr}}$ Provencher correspondit une politique linguistique peu exigeante, étant donné les difficultés éprou-

69 Dans son journal personnel, Mgr Olivier-Elzéar Mathieu, de Régina, fournit les réflexions suivantes quant au manque de prêtres d'origine anglaise dans son diocèse : "Ce qui rend l'exercice du saint ministère aussi ennuyeux que difficile, c'est qu'il n'y a presque pas de paroisse où le curé n'ait à parler qu'une seule langue. Il doit en savoir au moins deux, souvent trois ou quatre. ...

J'ai surtout de la difficulté à trouver des prêtres de langue anglaise. J'en ai demandé partout. D'abord, ils sont peu nombreux; puis ils n'aiment pas à apprendre d'autres langues que l'anglais ». Voir Saskatchewan Public Archives, "Journal de Mgr O.-E. Mathieu », Vol. I, p. 54. L'original est conservé aux Archives du Séminaire de Québec.

Deux autres confidences de Mgr Mathieu méritent qu'on s'y arrête. La première suivit une visite à Assiniboia en juin 1917 : «La paroisse renferme un plus grand nombre de français que d'anglais et ceux-ci voudraient avoir un prêtre de leur race. Ils sont toujours les mêmes partout! Ils sont chez eux au Canada, et tous les autres sont des étrangers qu'il faut négliger.» ibid., Vol. II, p. 4. Une visite épiscopale à Forget en juin 1918 poussa l'archevêque de Régina à confier à son journal une observation quelque peu déconcertance: "Dans cette paroisse [il $y$ a] une soixantaine de familles françaises et une vingtaine d'anglaises. Tout de même l'anglais a plus que sa part dans l'église. Il en est toujours ainsi dans les paroisses qui ont pour curés des prêtres venus de France et qui ont appris un peu d'anglais. Ces gens là tiennent moins à la conservation du français que les prêtres canadiens français. » ibid., pp. 42-43. 
vées dans le choix d'un clergé tout court. La population catholique du Nord-Ouest étant presque exclusivement française, il n'était pas nécessaire d'insister sur la connaissance d'une autre langue pour l'exercice du ministère. De toute façon, c'est sur place que l'on chercha à former des missionnaires disposés à évangéliser les peuples indigènes. Ce n'est que face à la concurrence des écoles protestantes dans les années 1840 que se posa le problème des écoles bilingues à opposer aux écoles unilingues anglaises.

Durant l'épiscopat de $\mathrm{M}^{\mathrm{gr}}$ Taché, la question des langues prit une nouvelle tournure. Celui-ci, résolu à respecter dans les faits la dualité française-anglaise qui avait été consacrée par des gestes constitutionnels et politiques en 1870, insista sur la nécessité d'un clergé bilingue, d'autant plus que le mouvement de population vers les terres fertiles de l'Ouest apportait d'importants groupements de colons qui n'étaient pas de langue française. C'est encore durant les dernières années de son épiscopat qu'il fallut songer pour la première fois à trouver des prêtres connaissant des langues autres que le français ou l'anglais.

L'époque la plus controversée fut sans contredit celle de 18961915. Les deux principaux chefs de l'Église dans l'Ouest, $\mathbf{M}^{\mathrm{gr}}$ Langevin de Saint-Boniface et $\mathrm{M}^{\mathrm{gr}}$ Legal de St-Albert, face à la vague d'immigration de nationalités étrangères au pays, tentèrent en premier lieu de satisfaire les besoins de ces peuples en leur envoyant un clergé français peu au diapason de leurs valeurs culturelles ou nationales. Ils se ravisèrent toutefois, non sans animosité de part et d'autre, et acceptèrent de reconnaître l'existence et les avantages de structures ecclésiastiques fondées sur des allégeances nationales. Ce fut l'époque du multilinguisme dans l'Ouest, et dans l'Église. Faut-il souligner les répercussions de cette politique sur les revendications des droits du groupe français à travers les trois provinces de l'Ouest, tant à l'époque que de nos jours?

Bref, s'il est une leçon à tirer des politiques linguistiques de l'Église de l'Ouest, c'est que l'universalisme de l'Église se devait de passer avant son penchant pour une nationalité quelconque, en l'occurrence celle du Canada français. Pouvait-il en être autrement?

Robert PAINCHAUD

Département d'histoire, Université de Winnipeg. 